\title{
Guest editorial to the special issue on MODELS 2009
}

\author{
Andy Schürr • Bran Selic
}

Published online: 5 April 2011

(C) Springer-Verlag 2011

The pioneering organizers of the first "UML" "98 workshop in Mulhouse, France in the summer of 1998 could hardly have anticipated that, in little over a decade, their initiative would blossom into today's highly successful MODELS conference series. MODELS is unquestionably the premier annual gathering of researchers and practitioners working on what is starting to emerge as a bona fide new technical discipline: model-based engineering of systems and software-a discipline distinguished by the fact that it fundamentally relies on the development and exploitation of models and supporting technologies.

It is fascinating to observe the progression of model-based technologies from early informal notations, supported by the now mostly forgotten "CASE" tools and intended as a kind of minor power assist for documenting analysis and design, to their present much more capable and more relevant state. Furthermore-as a number of the papers in this special issue amply demonstrate-we are finally seeing the gradual evolution of a systematic theoretical underpinning in support of these methods. Two types of forces are driving this progression: (1) the increasing realization that traditional codecentric methods are less and less capable of supporting the development of modern highly sophisticated systems and (2) the success of numerous industrial projects that have taken advantage of model-based engineering.

The MODELS conferences have contributed greatly to the heightened interest in the field, attracting the necessary young talent and providing a venue for reviewing both the state of

\footnotetext{
A. Schürr $(\bowtie)$

Technische Universität Darmstadt, Darmstadt, Germany

e-mail: andy.schuerr@es.tu-darmstadt.de

B. Selic $(\varangle)$

Malina Software Corp., Nepean, ON, Canada

e-mail: bran.selic@gmail.com
}

the art and the state of the practice. Consequently, the proceedings of the MODELS conferences represent a primary source for anyone undertaking a deeper study of model-based engineering.

The twelfth edition of the conference took place in Denver in the USA from October 4 through 9, 2009 along with numerous satellite workshops, tutorials as well as several other related scientific gatherings. The conference was exceptionally fortunate to have three high-quality invited keynote speakers and industry luminaries: Stephen Mellor, Larry Constantine, and Grady Booch.

A distinguishing feature of this edition of MODELS was the inclusion, for the first time, of a dedicated Empirical Results papers category. The objective was to provide a specific forum for publishing significant results, from both industry and research, of innovative application of modelbased methods in practice, so that the broader community could be better informed of the capabilities and successes of this relatively young discipline. Consequently, a significant proportion of accepted papers belong to this category, indicating an accumulation of pent up high-quality scientific and technical work which lacked a proper venue in the past and also demonstrating the increasing importance of modelbased approaches in practice.

The program committee received 248 submissions from 33 countries. A number of papers were co-authored by researchers from different institutions in different countries, indicating a very healthy trend towards scientific collaboration unhampered by institutional and geographic borders. Of these the committee selected 45 full papers and 13 short papers, for an acceptance rate of 18 and 5\%, respectively.

As is now customary, the head editors of the Journal of Software Systems and Modeling asked the programme committee to propose a selection of the best and most interesting papers from the conference for consideration to be published 
in a special issue. The authors of the selected papers were asked to expand on the original conference-paper content, to take advantage of the more extensive journal publication format, and also to supplement it with additional related research results. These extended contributions were then subjected to the standard peer review process and yet another selection, resulting in the collection of papers in this issue.

Pierre-Alain Muller, Frederic Fondement, Benoit Beaudry, and Benoit Combemale describe in their paper "Modeling Modeling Modeling" a reflective investigation into the essence of modeling itself-using modeling. They review current work on the theory of modeling and synthesize a metamodel that captures the different kinds of relationships that exist between models as well as between models and the realities they represent (which can, in some cases, also be models) on the basis of the purpose and nature of the models.

With the increasing interest in product line engineering, Gilles Perrouin, Gilles Vanwormhoudt, Brice Morin, Philippe Lahire, Olivier Barais, and Jean-Marc Jezequel provide a timely contribution that includes a novel use of aspectoriented methods to add variability into domain-specific modeling languages — as an aspect. Their paper, "Weaving Variability into Domain Metamodels", introduces a generic metamodel of variability and then describes how this metamodel can be integrated into existing metamodels, using techniques inspired by aspect-oriented approaches. This enables standardized treatment of variability in different languages that were not originally designed for that capability.

In their contribution, "Solving Dynamic Constraint Satisfaction Problems over Models", Ákos Horváth and Dániel Varró sketch how to build bridges between traditionally unrelated research communities that deal with constraint satisfaction problems over (static) models on one hand and the manipulation and modification of models by means of rulebased transformations on the other. They present a prototype solver for dynamic constraint satisfaction problems over models that relies on the basic capabilities of the model transformation environment VIATRA and its incremental pattern matching engine.

The paper, "Change-Driven Model Transformations: Change (in) the Rule to Rule the Change", by Gábor Bergmann, István Ráth, Gergely Varró, and Dániel Varró takes a dynamic approach to the problem of maintaining invariant relationships between models by focusing on the process of change in the source model and its consequences. It introduces a domain-specific language that supports a categorization of different kinds of model changes and rules for detecting and responding to them.

Shahar Maoz presents his work related to "Polymorphic Scenario-Based Specification Models: Semantics and Applications" that constitutes another step forward in the development and formalization of Life Sequence Charts (LSCs). LSCs are a variant of sequence diagrams with pre- cisely defined semantics that is simultaneously used for the scenario-based specification, testing as well as modeling-byexample-based implementation of object-oriented systems. Adding polymorphic concepts to LSCs based on behavioral subtyping increases their expressiveness and reusability significantly and closes a gap between the widespread usage of (subtype) polymorphism for modeling structural properties on one hand, but an ignorance of these concepts in behavioral OO modeling languages.

Finally, the paper "Concern Coverage in Base Station Development-An Empirical Investigation" by Lars Pareto, Peter Eriksson, and Staffan Ehnebom, takes us to the "practice" side of model-based engineering. It describes an empirical study of the use of model-based engineering methods and technologies in an industrial production setting. The study reveals that a large portion of important architectural-level concerns are still not addressed by current tools, languages, and practices, indicating that further foundational work and research are still outstanding.

\section{Author Biographies}

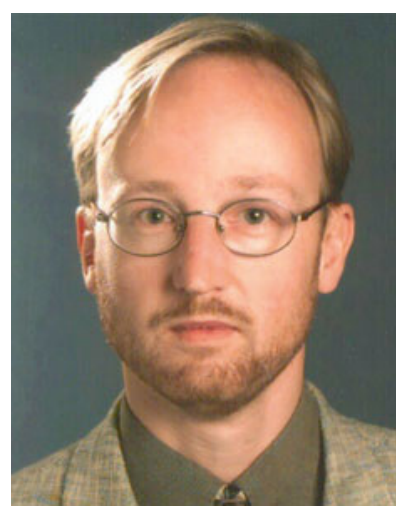

Andy Schürr holds the RealTime System chair of the Electrical Engineering and Information Technology Department of the Technische Universität Darmstadt. He is a member of the steering committees of the German GI Conference on Modeling, the European Conference on Model Foundations and Applications (ECMFA), the International Conference on Graph Transformations (ICGT), and the International ACM/IEEE Conference MODELS. His main research interests are model-driven engineering of embedded system software on one side and on the other side, the development of visual formal specification languages used for that purpose. His research group develops the modeling tool MOFLON that combines the OMG standards MOF and OCL with programmed/bidirectional graph transformation system technology.

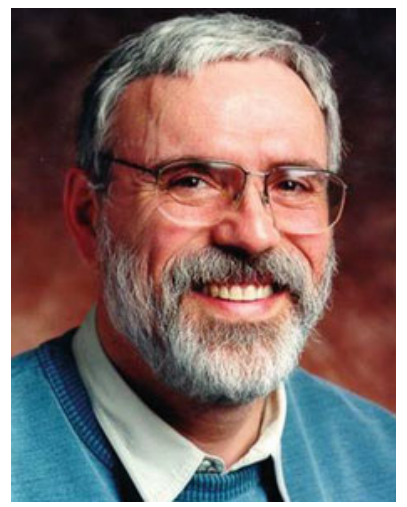

Bran Selic is President of Malina Software Corp. and also Director of Advanced Technology at Zeligsoft Limited in Canada as well as Visiting Scientist at Simula Research Labs in Norway. Prior to retiring in 2007, he was an IBM Distinguished Engineer responsible for defining the software tools strategy at IBM Rational. In 1992, Bran co-founded ObjecTime Limited a company which successfully pioneered the use of model-based tools and methods in the realtime embedded domain. He was very loosely involved in the definition and standardization of the UML modeling language. 